\title{
Chapter 11 \\ Discourses on Synthetic Biology \\ in Europe, India and China
}

\author{
Virgil Rerimassie, Dirk Stemerding, Wenxia Zhang \\ and Krishna Ravi Srinivas
}

\subsection{Introduction}

Synthetic biology constitutes a new field of research, in which scientists are gaining more and more control over the fundamental building blocks of life. This allows them to 'design' and 'create' microorganisms that may perform a variety of useful tasks, but at the same time become increasingly isolated from organisms we may find in nature. Given the potential of synthetic biology to contribute to addressing important challenges in, for example, health, the scarcity of resources and energy security, it is no surprise that this field has been embraced as a promising scientific endeavour by scientists all over the globe. On the other hand, like agro-biotechnology and nanotechnologies-or any other field of science and technology, for that matter-synthetic biology also gives rise to concerns about potential risks. In addition, it raises moral questions and concerns, since it allows scientists to put 'life' and 'nature' on the drawing board as never before.

\footnotetext{
V. Rerimassie $(\bowtie) \cdot$ D. Stemerding

Rathenau Instituut, P.O. Box 95366, 2509 CJ The Hague, The Netherlands

e-mail: v.rerimassie@ rathenau.nl

D. Stemerding

e-mail: d.stemerding @ rathenau.nl

W. Zhang

Chinese Academy of Science and Technology for Development (CASTED), 8 Yuyuantan South Road, Haidian District 100038, Beijing, China

e-mail: zhangwx@ casted.org.cn

K.R. Srinivas

RIS Research and Information System for Developing Countries, Zone IV-B, Fourth Floor, India Habitat Center, Lodhi Road, New Delhi 110 003, India

e-mail: ravisrinivas@ ris.org.in; krsriniv@gmail.com
} 
Synthetic biology may thus - once more, like any other science or technologyalso lead to tensions (and even conflict) and therefore debate. The nature and dynamics of these tensions and debates are, however, not solely informed by the character of a particular science or technology; they are also informed by the specific socioeconomic conditions, cultures and values in a given locality, which will therefore be taken into account in our comparative analysis of the three regions. Furthermore, we see synthetic biology as a global endeavour, contributing to an increased global interconnectedness. This is expressed, for instance, by increasing international scientific cooperation, but also by potential risks that are not constrained by state borders.

The contribution of synthetic biology to this increased global interconnectedness was recently underscored by the Global Network of Science Academies (IAP) (IAP 2014a). ${ }^{1}$ The IAP issued a statement appealing for global commitment regarding synthetic biology, recommending continuing worldwide collaboration between researchers and those regulating and enabling synthetic biology, and also calling for controversial issues to be settled. However, as we previously mentioned, moral concerns may lead to tension within a specific region, but also between different regions.

Against the backdrop of these region-specific traits and global interconnectedness, this chapter will analyse and compare the emerging debate on synthetic biology in the EU, China and India. The analysis will be based primarily on three reports of the Global Ethics in Science and Technology (GEST) project, each focusing on discourses on synthetic biology in the region concerned (Stemerding and Rerimassie 2013; Zhang 2014; Srinivas 2014). We consider the debate in the EU rather mature in comparison with those in the other regions, and moreover believe that it reflects many traits of the international debate on synthetic biology. We will therefore use the European debate as a starting point, and then highlight region-specific traits in China and India.

In conducting this analysis we will use the analytical framework described in Chap. 8, thus analysing discourses on innovation, risk, and power and control. In addition, we will focus on two crosscutting discourses: first, public debates expressing lay morality — what expectations and issues have been raised concerning synthetic biology by voices from civil society and the broader public? - and second, how reflective ethics voices have engaged with synthetic biology in the three regions. We will examine the nature of each discourse: that is, what kinds of issues are discussed and to what values do they relate? What kinds of actors take part in the discussion? Then we will consider whether certain discourses are dominant. This will allow a comparative analysis of the three regions, which will consider similarities and differences.

\footnotetext{
1 The Global Network of Science Academies consists of 106 scientific academies from all over the world, including the EU, China and India (IAP 2014b).
} 
Throughout this endeavour we will pay specific attention to the role of regionspecific values, as described in Chaps. 5, 6 and 7, in the debate. We will begin, however, by briefly describing the understanding, framing and state of the art of synthetic biology in the three regions, since its understanding and the degree of development are highly likely to be core parameters for the nature, and even emergence, of the different discourses in the three regions.

\subsection{Understanding Synthetic Biology}

The theoretical basis of the contemporary understanding of synthetic biology is attributed largely to Waclaw Szybalski, who proclaimed in 1974:

Up to now we are working on the descriptive phase of molecular biology. ... But the real challenge will start when we enter the synthetic biology phase of research in our field. We will then devise new control elements and add these new modules to the existing genomes or build up wholly new genomes. This would be a field with unlimited expansion potential and hardly any limitations to building 'new better control circuits' and ... finally other 'synthetic' organisms ... (EGE 2009). ${ }^{2}$

Thus, according to Szybalski, biology will eventually evolve into a different kind of science, in which we shift from describing to designing, or redesigning, life. Szybalski's words have turned out to be prophetic, but it was not until the turn of the century that scientists started research under the explicit heading of synthetic biology. Interestingly, many of these researchers were not primarily involved in molecular biology. Today 'synthetic biologists' employ a variety of novel approaches, all of which allow increasing control over the fundamental building blocks of life. This unique quality can therefore also be found in the definitions used in the three regions to describe synthetic biology. ${ }^{3}$ A definition by a highlevel expert group for the European Commission (NEST 2005), for instance, reads as follows:

Synthetic biology is the engineering of biology: the synthesis of complex, biologically based (or inspired) systems, which display functions that do not exist in nature. This engineering perspective may be applied at all levels of the hierarchy of biological structures-from individual molecules to whole cells, tissues and organisms. In essence, synthetic biology will enable the design of 'biological systems' in a rational and systematic way.

\footnotetext{
${ }^{2}$ Luis Campos demonstrates that the label 'synthetic biology' can actually already be traced back to the beginning of the 20th century. The earliest explicit reference to 'synthetic biology' comes from the book La biologie synthetique by Stéphane Leduc (1853-1939) (Campos 2009).

${ }^{3}$ For a more detailed account of dominant approaches in synthetic biology see Stemerding and Rerimassie (2013).
} 
The Indian Task Force on Synthetic and Systems Biology Resource Network produced the following, quite similar, definition:

Synthetic biology refers to both:

- the design and fabrication of biological components and systems that do not already exist in the natural world; and

- the re-design and fabrication of existing biological systems (SSBRN 2012).

In the official China Biotechnological Development Report, synthetic biology is described as:

... a new trend of biotechnological development ... to form new biological systems and achieve expected industrial application (Department of Science and Technology for Social Development under the Ministry of Science and Technology of China, China National Center for Biotechnology Development 2009).

Synthetic biology is thus commonly understood as the application to biology of a true engineering approach, in order to design, or redesign, organisms that are useful for society.

\subsubsection{Framing of Synthetic Biology as an Emerging and Converging Technology}

The emergence and introduction of new science and technology in society are, more often than not, accompanied by tensions and conflicts. However, as a new and emerging field of engineering, synthetic biology is still largely at a laboratory stage. Therefore discussions about synthetic biology as a potential source of tensions and conflicts will be strongly influenced by experiences with other science and technology developments in the recent past:

To debate a still quite abstract technology, participants functionally need a frame that determines which arguments are legitimate and which issues are relevant (Torgersen and Schmidt 2013).

According to Torgersen and Schmidt, three fields of science and technology currently provide important frames for discussing synthetic biology: (green) biotechnology, nanotechnology and information technology. 4 This makes even more sense, because synthetic biology is often considered to be enabled by so-called 'NBIC convergence': the synergetic convergence of nanotechnologies, biotechnologies, information and communications technologies and, though less relevant in this context, cognitive sciences (Van Est and Stemerding 2012).

\footnotetext{
4 'In the biotechnology debate, risk has long been emphasised over economic benefits. More recently, nanotechnology has been referred to mostly in terms of benefits, while risks tended to be an issue for scientific discourses. This has frequently been related to the many outreach activities around nanotechnology. Information technology, finally, has retained the image of being “cool” and useful on a personal level' (Torgersen and Schmidt 2013).
} 
When looking at the three regions, we must conclude that synthetic biology is predominantly framed, and debated, as a new phase in the development of biotechnology, as illustrated in statements by important spokespersons in the field. According to the New and Emerging Science and Technology High-Level Expert Group for the European Commission (NEST 2005), synthetic biology could revolutionize the biotechnology industries and perhaps even biology as a science. In important symposiums, such as those organized by the China Association for Science and Technology, synthetic biology is described by Chinese biologists as one of the great frontiers of modern biotechnology. The Indian task force sees synthetic biology as a science of the future, which may change the profile of the biotechnology industry. Synthetic biology is thus commonly understood in the three regions as the next wave in biotechnology, which will also strongly frame societal debates about its potential implications. Conceived as a new form of 'extreme genetic engineering', synthetic biology may well add fuel to the ongoing debates and controversies surrounding genetically modified organisms (ETC Group 2007).

\subsection{The Development of Synthetic Biology in the Three Regions: The State of the Art}

Ever since the turn of the century, synthetic biology has been gaining international momentum and the number of published papers has been steadily increasing (Zhang 2014). As illustrated in an interactive map (reproduced in Fig. 11.1) produced by the Synthetic Biology Project, which is led by the US-based Woodrow Wilson International Center for Scholars, synthetic biology research activity has emerged all over the globe. In addition, there is increasing international

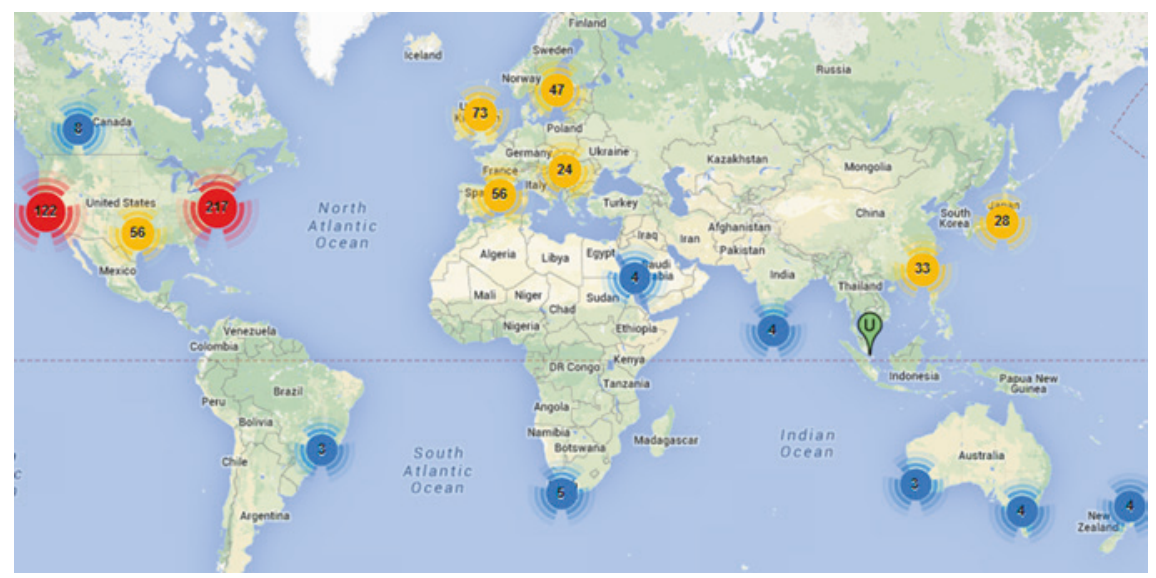

Fig. 11.1 Map tracking the number of synthetic biology research groups across the globe (Reproduced from http://www.synbioproject.org/library/inventories/map/) 
collaboration among synthetic biologists, contributing to an ever growing global interconnectedness.

There are significant differences across the three regions in the development of synthetic biology, which ranges from rather advanced to just starting. In Europe such development is not quite as advanced as in the United States, but is still very much at the forefront. Once synthetic biology emerged in the United States, it was almost immediately embraced by the European scientific community, and the EU (as well as several individual EU member states) rapidly started investing in synthetic biology as well (Stemerding and Rerimassie 2013). By 2014, synthetic biology was gradually being applied in industrial settings (Schmidt 2012).

Synthetic biology has also attracted the attention of Chinese researchers, although this did not begin happening as early as in Europe. The Chinese government started funding synthetic biology research in about 2008, and ever since has given the field more and more support. The development of synthetic biology in China is therefore not as advanced as in Europe, but now the country is fully equipped to catch up with countries at the forefront (Zhang 2014).

In sharp contrast, synthetic biology has so far gained little attention in India, and is as yet largely confined to certain institutes and groups-very few in comparison with the number of Indian groups working in life sciences and biotechnology. Also the interest from the Indian government and industry is limited so far (Srinivas 2014). Accordingly, this chapter's discussion of the discourses on synthetic biology in India will be primarily based on the findings of the aforementioned task force, made up of government representatives and academics, which was established by the government to examine the opportunities for synthetic biology and systems biology for India.

\subsection{Comparing Discourses on Synthetic Biology in the Three Regions}

Preceding chapters have described dominant approaches in synthetic biology and observed that in all three regions the field is understood as a new phase in the development of biotechnology. It has also been noted, however, that the state of the art of the development of synthetic biology differs widely among the three regions: development in the EU can be seen as 'advanced', in China as 'rapidly catching up' and in India as 'just starting'. Against this backdrop, we will now analyse and compare how synthetic biology has been debated so far in the three regions, based on discourses relating respectively to innovation, to risk, and to power and control, and also those concerning lay morality and reflective ethics. We will highlight the issues being discussed, the values to which they relate and the actors that play a part in the discourse. A more detailed account of the discourses in each region can be found in the three GEST reports on this topic (Stemerding and Rerimassie 2013; Zhang 2014; Srinivas 2014). 


\subsubsection{Discourses on Innovation}

Why is synthetic biology important? What are the opportunities? What can the field deliver? What is needed for synthetic biology to mature into an industrially relevant and socially robust discipline? These are the central questions of an innovation discourse.

In all three regions voices are heard on the opportunities synthetic biology might bring for society. It is perceived as a potentially powerful field of research whose applications might help address challenges that all three regions face. Such challenges may relate to (public) health, sustainability, energy sources and ecology. Synthetic biology is also seen as a vital source of future economic development in the context of global competition between the three regions. Thus support for synthetic biology should enable China to 'catch up' with the US and Europe and is called for in India so that the country does not 'miss the bus' (Zhang 2014; Srinivas 2014).

In Europe, the New and Emerging Science and Technology (NEST) Pathfinder funding scheme, set up in 2004, was an important starting point for the development of synthetic biology (Pei et al. 2011). It was followed by several other initiatives, including those under the Seventh Framework Programme and the road map of the project called Towards a European Strategy for Synthetic Biology (TESSY), which is dedicated to strategy development for synthetic biology in Europe (TESSY 2008; Gaisser et al. 2009). More recently, from 2012 to 2014, came the establishment of ERASynBio, a European initiative aimed at the development and coordination of synthetic biology in the European research area. One of the important aims of this initiative was to comprehensively map national and transnational funding programmes, funded synthetic biology projects, relevant strategies and reports, and active companies, in order to develop a strategic vision. According to these mapping activities, about $€ 450$ million of public research funding was allocated to synthetic biology from 2004 to 2014 (ERASynBio 2014) (Fig. 11.2).

Important issues that need to be addressed, according to the ERASynBio network, include building a transnational multidisciplinary research community, data sharing, standardization and accelerating the applied and industrial use of synthetic biology. Another particularly important issue is what a proper intellectual property regime for synthetic biology would look like. Should it allow broad patenting or would an open-source regime be more desirable? As for values, we find that the European discourse is predominately informed by values of freedoms, including market freedoms, and sustainability (e.g. EASAC 2010). Furthermore, in the EU a need is felt to go beyond technoscientific support actions in order to make synthetic biology successful, for instance by addressing ethical and regulatory concerns early on (e.g. TESSY 2008; OECD, Royal Society 2010). This is aptly expressed in a broadly shared call for responsible research and innovation (RRI) in synthetic biology (e.g. ERASynBio 2014). Interestingly, in considering the innovation potential of synthetic biology, the Indian task force also explicitly mentioned issues of risk and ethics as equally important to address. 
Fig. 11.2 Sites of major European public investment in synthetic biology (ERASynBio 2014)

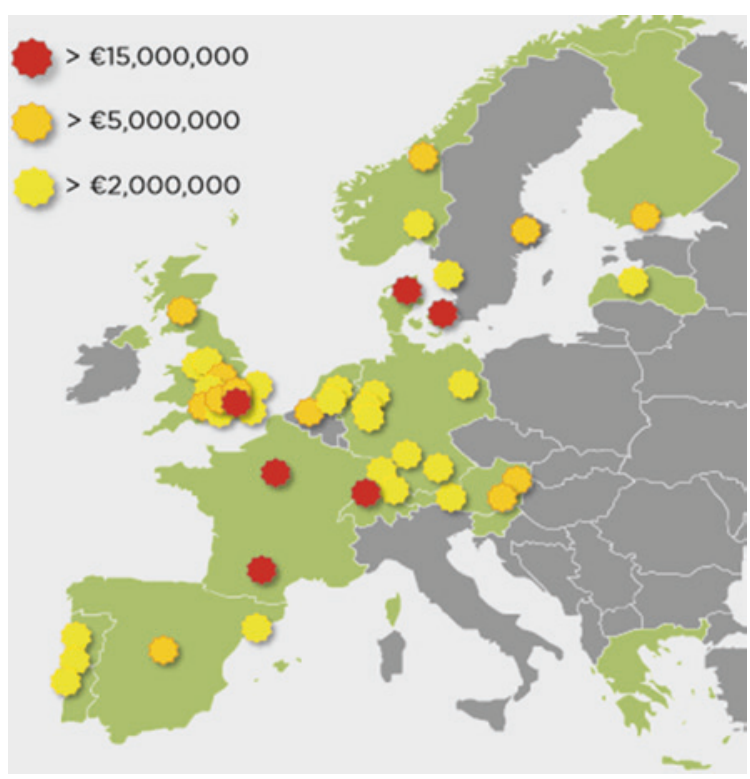

As in Europe, there is in China a strongly developed innovation discourse, from which several support actions are apparent, such as capacity and community building (Zhang 2014). In many scientific symposia, such as the Xiangshan Science Conference sessions, it is evident that Chinese biologists consider synthetic biology to be one of the great frontiers of modern biotechnology. These biologists have therefore called for government support on many occasions, and not without success. For example, synthetic biology is marked as one of the 12 core key technologies for prioritized development in the 12th Five-year Plan for Biotechnological Development (MOST 2011) released by the Ministry of Science and Technology. Furthermore, synthetic biology has been financed by a series of programmes, such as the 863 programme, ${ }^{5}$ the 973 programme ${ }^{6}$ and the National Natural Science Foundation.

Regarding values, the innovation discourse is informed by the full spectrum of Chinese values. Stimulating synthetic biology is expected to contribute to promoting economic development (progress), protecting public health (harmony), safeguarding national security and coping with an ageing and growing population (peace), and last, addressing resource and environmental needs (sustainability) (CNCBD 2010). This illustrates that while ethics is not institutionalized in China

\footnotetext{
5 The 863 programme, or the National High-tech R\&D Programme, was approved in 1986 to promote high-technology research and development in China. Biotechnology is listed as one of its eight priority fields.

6 The 973 programme, or the National Basic Research Programme of China, was approved in 1997 to support basic science and technology research. It promotes research and innovation in fields of far-reaching and strategic importance, such the life sciences.
} 
in the same way as it is in the EU or the US, it certainly has a place in directing the course of synthetic biology in China.

India, unlike Europe and China, does not have a strongly developed innovation discourse yet. In the recommendations of the Indian task force, however, there is a strong link between the identified potential aims for synthetic biology in India and the needs of the country, ranging from energy security to improvements in agriculture and health, topics that strongly relate to values of access and equity.

Last, in all three regions the International Genetically Engineered Machine competition (iGEM), a synthetic biology design contest for students, is considered an important educational, capacity-building and community-building tool (see e.g. ERASynBio 2014; SSBRN Task Force 2012; Zhang 2014).

\subsubsection{Discourses on Risk}

Like any other technology, synthetic biology not only promises potential benefits, but also raises concerns about possible risks. What types of risks are perceived? By whom? What weight do they assign to the risks in relation to the benefits? How should society deal with these potential risks?

The potential risks of synthetic biology make up an important part of the evolving story of synthetic biology. This is the case for the EU, but also for China and India. Two types of categories of risks are distinguished: first, biosafety, relating to potential unintended consequences for humankind and the environment, and second, biosecurity, relating to potential misuse (e.g. EGE 2009; CNCBD 2010; SSBRN Task Force 2012). The fact that all three regions devote attention to these issues is no real surprise, however, since EU member states, India and China are all party to several international conventions, such as the Cartagena Protocol on Biosafety and the Biological Weapons Convention, which call for these issues to be addressed as well.

Civil society organizations in Europe, unlike the other regions, are strongly involved in the evolving risk discourse. A central value in the European discourse is protection as a citizen's right. In terms of this right, civil society organizations demand a strict precautionary approach and also bring in a more inclusive perspective of justice, solidarity and equality. Whereas synthetic biology is supported in the innovation discourse for its potential contribution to a greener economy, civil society organizations challenge it for its detrimental effects on sustainability; that is, for promoting the exploitation of natural resources and the communities that are dependent on them (FOE et al. 2012). Indeed, similar concerns have been voiced in India (Srinivas 2014).

In China risks are also seen as a point of concern, but hampering innovation and missing out on the opportunities of synthetic biology are actually perceived as much bigger risks by both the government and the scientific community. This became clear, for instance, at an academic symposium dedicated to synthetic biology and ethical and biosecurity concerns, organized by the China Association 
for Science and Technology in 2010. In fact, some experts, such as the Chinese scientist Yang Huanming, expressed their concern about the influence of critical public opinion in the developed countries on public perceptions in China and called for positive publicity for synthetic biology (Yang 2011). Another Chinese scientist, Du Lin, advocated efforts to create a consensus that the discussion of ethical and biosafety issues relating to synthetic biology should not hinder synthetic biology research in China. In his view, such a discussion should serve the purpose of responding to future international opposition, rather than hindering China's development in this field of research (Du 2011). Progress therefore seems to be dominant value in the Chinese risk discourse.

\subsubsection{Discourses on Power and Control}

On one hand, synthetic biology may provide opportunities to address the grand challenges societies are facing, including those relating to health, energy and sustainability. On the other hand, synthetic biology may give rise to risks and ethical concerns. In a response to this tension, a recent statement by the Global Network of Science Academies called for a global commitment regarding synthetic biology (IAP 2014a). According to IAP chairman Volker ter Meulen, it is time to settle the 'synthetic controversy' (ter Meulen 2014). He notes that if synthetic biology is to thrive, the world needs to decide now how the field should be regulated and supported. In a comment in The Guardian, science and technology studies scholar Jack Stilgoe (2014) responded:

My question is why we, the public, are shut out of the conversation about benefits.

'Realising the potential' of SynBio is talked about as though that potential is pre-ordained.

It isn't. SynBio will become what scientists, innovators, users, regulators and others make of it. It could be used to create brilliant, emancipatory, subversive, public-value innovation, or it could bolster existing power structures. The direction will depend on who is involved, what they value, what research gets done, how intellectual property (IP) is arranged and more.

So who gets to decide the direction in which synthetic biology should develop and under what conditions? In other words: who gets to exercise the power and control over synthetic biology?

Our case studies found a strongly developed discourse on this topic only in the EU. The main actors are government and the scientific community, but civil society is also making its mark (ETC Group 2007; Roco 2008; Tait 2009; IRGC 2010; EASAC 2010; Zhang et al. 2011; FOE et al. 2012; ERASynBio 2014). Largely inspired by earlier bad experiences with the public reception of technologies, both policymakers and the scientific community feel the need to involve stakeholders and the broader public early in the development of synthetic biology. The aim is that such involvement, besides addressing ethical, legal and social issues head on, allows synthetic biology to be better embedded in society, which resonates well with the European striving towards responsible research and innovation 
(EC 2013). In order to foster responsible research and innovation in the field of synthetic biology, the European Commission for instance funded the Synenergene programme (2013-2017), which is dedicated to responsible research and innovation and public engagement in synthetic biology (Synenergene 2014). Furthermore, European policymakers and the scientific community call attention to issues that require a delicate balance, such as devising forward-looking regulation without stifling innovation and maintaining the equilibrium between scientific freedom and self-regulation vis-à-vis state-driven regulation and coercion. This latter form of hard government is particularly advocated by internationally operating NGOs such as Friends of the Earth and the Action Group on Erosion, Technology and Concentration, or ETC Group. As a result, the European governance landscape of synthetic biology is already rather complex, even while synthetic biology is still predominantly confined to the laboratory.

Turning to the Indian case, the Indian task force also recommends that ethical, legal and social implications should be addressed and public opinion mapped upfront, rather than technological development alone being stimulated as a top priority. To substantiate this stance, it refers to the negative consequences of prematurely pushing biofuels. In addition, the task force raises the tension between open-source initiatives and rigid intellectual property approaches, as these relate to the Indian values of access, equity and inclusion.

The Chinese discourse shows a different picture, namely that the development and management of synthetic biology are largely in the hands of the government and the scientific community, and are considered sufficient so far (Zhang 2014).

\subsubsection{Synthetic Biology and Lay Morality}

Public reception is crucial for the course of development of a technology. To put it bluntly: it can make or break a technology. The concerns of members of the public may involve potential physical harms, but to a large extent they will also relate to non-physical issues: that is, boundaries related to their values and culture that should not be overstepped.

However, given its early stage of development, synthetic biology has not yet given rise to significant public debate. In fact, even public awareness is rather low. ${ }^{7}$ In the EU there is no real active debate so far, apart from the voices of a small number of NGOs, but surveys among the general public and organized public dialogues already demonstrate a large degree of pluralism, involving a variety of issues and values (RAE 2009; Battachary et al. 2010; Rerimassie and Stemerding 2014; EC 2010). Such issues include concerns regarding biosafety and biosecurity,

\footnotetext{
7 Only in the EU has public awareness of synthetic biology been gauged via surveys. The 2010 Biotechnology Eurobarometer (EC 2010) revealed that a large majority of EU citizens (83\%) had never heard of synthetic biology.
} 
freedom of research, monopolization and increasing global inequalities, relating to a broad spectrum of values, such as citizens' rights and market freedoms, justice, solidarity, equality and sustainability. These issues and values will prove difficult to reconcile. This is sure to become a serious challenge for the governance of synthetic biology, given the aspiration to include a wide array of actors and the issues they put forward.

China has not seen an active debate on synthetic biology so far. The public hold science in high esteem and trust the government's management of synthetic biology. This attitude reflects the Chinese value system, in which, in the pursuit of progress and affluence, pragmatism and developmentalism prevail over potential risks as long as the latter have not materialized as actual threats (Zhang 2014). This fits in well with the findings on Chinese public perceptions of science and technology (See Chap. 3).

Unsurprisingly, there is no public debate on synthetic biology in the Indian case either. However, a well-known opponent of genetic modification, Vandana Shiva, recently voiced criticism of synthetic biology, as did certain environmental groups in India (Domicone 2013). The concerns raised mostly relate to socioeconomic considerations and values such as equity and access.

\subsubsection{Synthetic Biology and Reflective Ethics}

In addition to public expressions of lay morality, morality is reflected upon by voices in the field of reflective ethics. Such voices may belong to academia or to ethics advisory bodies, including groups and organizations engaged in technology assessment and the examination of ethical, legal and social (ELSI) implications. Have such voices been heard so far in the emerging debate on synthetic biology? If so, are they making an impact on science and technology policy-making and development?

In the EU there is strong involvement by reflective ethics voices. In fact, the European technology assessment and academic communities engaged with synthetic biology early (Douglas and Stemerding 2014). The EU has contributed substantially to this effort by funding several programmes on ethical, legal and social implications, such as SYNBIOSAFE, Synth-Ethics and SYBHEL (for Synthetic Biology for Human Health: Ethical and Legal Issues). On one hand, reflective ethics voices analyse and deepen issues and concerns that have already been raised by other actors. On the other hand, reflective ethics also enriches debate by addressing issues that so far have not played a big part, but might in future. A good example is an in-depth analysis of the notion of 'playing God' conducted by the EU project SYNTH-ETHICS (Link 2011). This notion is considered potentially controversial in the context of synthetic biology since it revives concerns that have been voiced regarding earlier biotechnology as well.

Another important contribution was made by the European Group on Ethics, which gave a detailed account of EU regulations and the relevant global provisions 
with regard to biosafety, biosecurity, intellectual property and potential applications of synthetic biology, and also the international framework of ethics and human rights (EGE 2009). On the basis of this international human rights framework, the European Group on Ethics articulates the main values that should guide the ethics of synthetic biology, including human dignity, autonomy and responsibility, freedom, equality, solidarity, justice and sustainability—indeed, largely corresponding with the spectrum of European values described in Chap. 5. Broadly speaking it is possible to identify four different roles that reflective ethics is playing in the emerging debate on synthetic biology:

- Articulating values and issues

- Highlighting tensions (often hidden) between values

- Enriching debate

- Translating the articulated values and issues into science and technology policy-making

It is perhaps not surprising that such efforts are being made in Europe, since earlier biotechnologies aroused considerable controversy.

In China some reflective ethics voices from academia are heard, but systematic reflection on the moral aspects of synthetic biology is generally lacking. In fact, ethical reflection has so far mainly been limited to the general introduction and citing of foreign views. A few ethics scholars have raised their voice in an appeal for issues of risk and ethics to be addressed in more 'authentic' ways by surveying the important philosophical and cultural factors in Chinese public policy-making concerning synthetic biology (Zhai and Renzong 2010). Given China's social and cultural environment and its prevailing pragmatism and developmentalism, little public resistance against synthetic biology is expected (Zhang 2014).

In India, too, no clearly visible tradition of reflective ethics can be found, but the Indian task force does recommend that ethical issues be addressed in a an 'atmosphere of public acceptance and transparency', given potential sensitivities raised by synthetic biology as a science that interferes with life (Srinivas 2014).

\subsection{Conclusion and Discussion: Governance Challenges}

This chapter has set out to map the evolving debates on synthetic biology in the EU, China and India. It has demonstrated that the European debate on synthetic biology has already become quite mature, the debate in China is taking form, and the Indian debate is slowly emerging (Table 11.1).

Comparing the nature of the debates in the three regions reveals distinct features and positions on the pros and cons of synthetic biology. On one hand, these debates are informed by differences in the state of the art of synthetic biology in the respective regions. On the other, they are informed by region-specific socioeconomic conditions, cultures and value systems. Based on our analysis we have identified a number of region-specific governance challenges that will play an 
Table 11.1 Overview of synthetic biology discourses in China, the EU and India (shading of cells indicates degree of development)

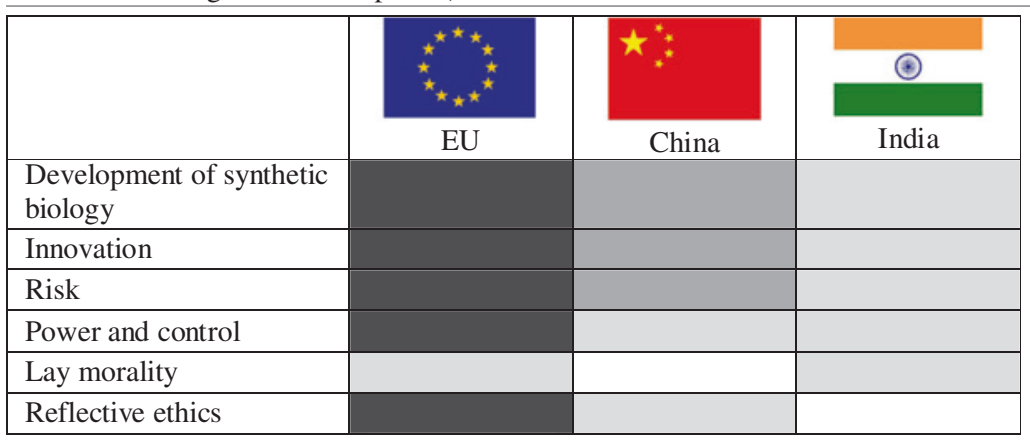

important role in the evolution of the debates on synthetic biology in the three regions. These challenges are summarized in Table 11.2.

To better understand these governance challenges we should distinguish between risk governance and innovation governance. Discourses of risk in the three regions are being framed by scientific knowledge and legal regimes that are negotiated and established not only in a national or regional context, but also a global context. In other words, risk governance has a strong international dimension. In all three regions we have seen that, in discussions of synthetic biology, attention is drawn to issues of biosafety and biosecurity, articulating the need for governments to match global standards of regulation laid down in international agreements, such as the Cartagena Protocol on Biosafety and the Biological Weapon Convention. This global interconnectedness also creates international forums in which parties can search for common ground on how to deal with biosafety and biosecurity risks in synthetic biology.

In our understanding of innovation governance in the three regions, a global perspective is also obviously important, given the dynamics of international competition, exchange and cooperation. At the same time, innovation governance in the three regions relates to socioeconomic and socioethical issues that are more specifically framed by regional contexts, values and concerns. In Europe, synthetic biology is basically funded as 'blue sky' research in support of market-driven development. In China and India one finds more deliberate attempts at priority setting in the framework of governmental five-year plans. The Chinese government has identified synthetic biology as a technology to be prioritized strategically in the nation's applied biotechnological research, especially in the biomedical and health care field. The Indian task force synthetic biology report put the emphasis on meeting the developmental needs of the country, and identified biofuels as one of the key applications.

In these different regional contexts of synthetic biology innovation, the role of the public and reflective ethics also differs, raising governance challenges that are specific for each region (as indicated in Table 11.2). In Europe, as we have seen, experts have emphasized the need to address ethical and safety concerns 


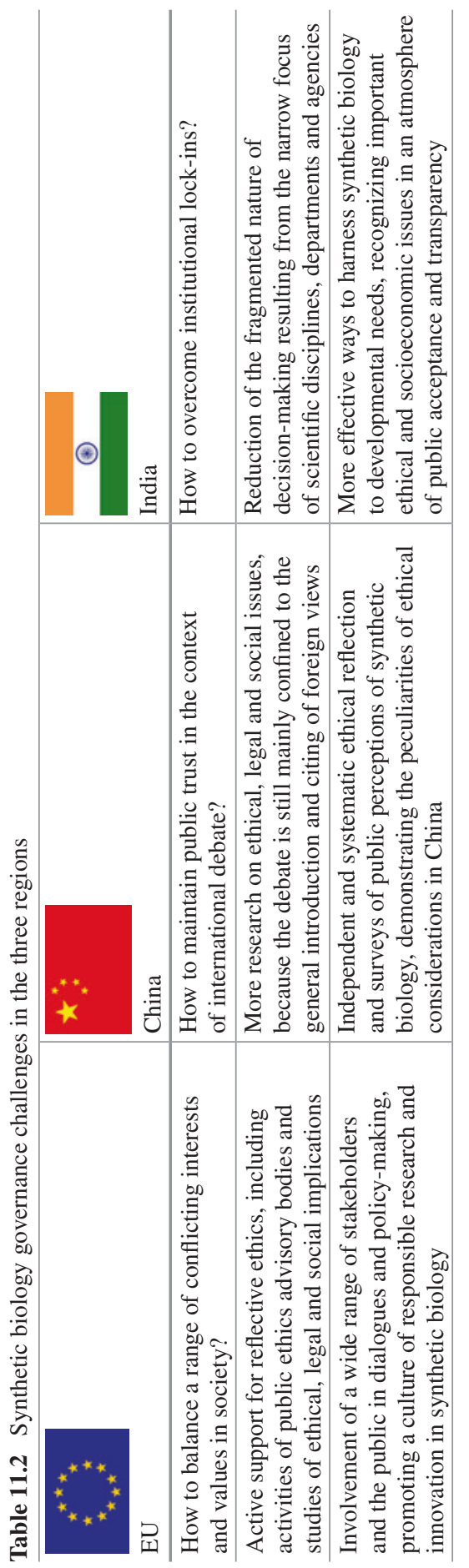


from the very beginning. Studies of the ethical, legal and social implications have received active support, and there have been several public dialogue initiatives about synthetic biology. The resulting challenge for the European governance of synthetic biology is how to balance the range of interests and values of all relevant stakeholders.

In China the government is the principal agent in synthetic biology policymaking, and there has not been much demand for control from scientists or the public. The public generally hold science in high esteem, and systematic ethical reflection on synthetic biology is mostly lacking. However, there is clear concern among scientists that public opinion might be swayed by critical accounts of synthetic biology in the foreign media. How to maintain public trust in the context of international public debate thus arises as a key governance challenge in China.

In India, the task force took a broad view of the promotion and regulation of synthetic biology, emphasizing its potential benefits but also the need to address safety and ethical issues and to take the public into account. However, conventional patterns of budget allocation and established institutional divisions in India are not conducive to the development of a broad interdisciplinary field and to effectively directing synthetic biology innovation to the country's socioeconomic needs.

What are the implications of these governance challenges for our aim to strengthen 'global ethics' in science and technology policy-making in a world of increasing interconnectedness? Our case study demonstrates that issues articulated in public and ethics discourses about synthetic biology are strongly framed by debates emerging in a European and American context. Indeed, to the extent that these issues are addressed in the Chinese or Indian regional context, they often reflect or replicate European public and ethics discourses rather than more specific regional values and concerns.

A truly global dialogue will therefore require a strengthening of public and ethics voices in all three regions, in ways that respond to the specific governance challenges in each region and more specifically reflect regional values and concerns. Such a dialogue will also require a global forum to support mutual learning about the possibilities and implications of synthetic biology on the basis of international exchange and cooperation. We have identified two forums that may play an important role in this regard. First, related to the world of synthetic biology innovation, we would like to highlight iGEM, the annual International Genetically Engineered Machine competition, which attracts student teams from all over the world. In just 10 years iGEM has developed into a global hub for thousands of young scientists to 'meet and compete' (Zhang et al. 2011). This global community not only offers a fascinating learning environment, it has also promoted an interest in synthetic biology among researchers in China, and could do so in India as well. Moreover, and most importantly for a global ethics in synthetic biology, iGEM requires teams to include in their projects 'policy and practices' work, which stimulates a global exchange and dissemination of ideas about biosafety and biosecurity, questions of intellectual property, ethics and public engagement in the emerging field of synthetic biology. Second, from the perspective of international public policy making, 
we see an important role for the United Nations Educational, Scientific and Cultural Organization (UNESCO). Over the course of time, UNESCO has been manifesting itself more and more as an important global ethics forum. In 2005 the general conference of UNESCO pioneered in this regard by acclamation of the Universal Declaration on Bioethics and Human Rights. For the first time in history, such a large number of UN member States committed themselves and the international community to respect and apply fundamental principles of bioethics set forth within a single document (UNESCO 2006). Therefore, we consider this an excellent opportunity to build on in dealing with emerging science and technology.

Open Access This chapter is distributed under the terms of the Creative Commons Attribution Noncommercial License, which permits any noncommercial use, distribution, and reproduction in any medium, provided the original author(s) and source are credited.

\section{References}

Battachary D, Calitz JP, Hunter A (2010) Synthetic biology dialogue. Biotechnology and Biological Sciences Research Council, Swindon

Campos L (2009) That was the synthetic biology that was. In: Schmidt M, Kelle A, GanguliMitra A, De Vriend H (eds) Synthetic biology: the technoscience and its societal consequences. Springer, New York, Heidelberg, pp 5-22

CNCBD (2010) China's biological economy: biotechnology and bio-industry innovation from the perspective of international comparison. China National Center for Biotechnology Development. China Agricultural Science and Technology Press, Beijing

Department of Science and Technology for Social Development under the Ministry of Science and Technology of China and China National Center for Biotechnology Development (2009) China biotechnological development report 2008. Science Press, Beijing, pp 128-130

Domicone A (2013) Synthetic biology: an emperor with no clothes? PMJWire, Policy Matters Journal. http://www.policymattersjournal.org/2/post/2013/09/-synthetic-biology-an-emperorwith-no-clothes.html. Accessed 2 June 2014

Douglas CMW, Stemerding D (2014) Challenges for the European governance of synthetic biology for human health. Life Sci Soc Policy 10(2):1-18

Du L (2011) Ethical issues and biosafety goals of synthetic biology explained. Academic division of China association of science and technology. Ethical issues of synthetic biology and biosafety. China Science and Technology Press, Beijing, pp 26-28

EASAC (2010) Realising European potential in synthetic biology: scientific opportunities and good governance. German Academy of Sciences Leopoldina, Halle

EC (2010) Special Eurobarometer 340: science and technology. European Commission, Brussels

EC (2013) Options for strengthening responsible research and innovation. Report of the Expert Group on the state of art in Europe on responsible research and innovation. European Commission, Brussels

EGE (2009) Ethics of synthetic biology. Opinion no. 25. European Group on Ethics, European Commission, Brussels

ERASynBio (2014) Next steps for European synthetic biology: a strategic vision from ERASynBio. European research area network for the development and coordination of synthetic biology in Europe

ETC Group (2007) Extreme genetic engineering: an introduction to synthetic biology. ETC Group, Ottawa 
FOE, Cta, ETC Group (2012) The principles for the oversight of synthetic biology. Friends of the Earth, Washington, DC

Gaisser S, Reiss T, Lunkes A et al (2009) Making the most of synthetic biology: strategies for synthetic biology development in Europe. EMBO Rep 10(Suppl 1):S5-S8

IAP (2014a) IAP Statement on realising global potential in synthetic biology: scientific opportunities and good governance. Global Network of Science Academies, Trieste

IAP (2014b) Academies. http://www.interacademies.net/Academies.aspx. Accessed 2 June 2014

IRGC (2010) Guidelines for the appropriate risk governance of synthetic biology. International Risk Governance Council, Geneva

Link H (2011) In-depth analysis of outstanding philosophical issues in: SYNTH-ETHICS Report WP1: identification of ethical issues and analysis of public discourse, pp 40-49

MOST (2011) 12th five-year plan for biotechnological development. Ministry of Science and Technology of the People's Republic of China, Beijing

NEST (2005) Synthetic biology: applying engineering to biology. Report of a NEST high-level Expert Group. Office for Official Publications of the European Communities, Luxembourg

OECD, Royal Society (2010) Symposium on opportunities and challenges in the emerging field of synthetic biology. OECD and the Royal Society, London

Pei L, Schmidt M, Wei W (2011) Synthetic biology: an emerging research field in China. Biotechnol Adv 29(6-3):804-814. doi:10.1016/j.biotechadv.2011.06.008

RAE (2009) Synthetic biology: scope, applications and implications. Royal Academy of Engineering, London

Rerimassie VG, Stemerding D (2014) SynBio politics: bringing synthetic biology into debate. Rathenau Instituut, The Hague

Roco M (2008) Possibilities for global governance of emerging technologies. J Nanopart Res 10:11-29

Schmidt M (ed) (2012) Synthetic biology. Industrial and environmental applications, WileyBlackwell, Weinheim, Germany

Srinivas KK (2014) The status of synthetic biology in India. In: Stemerding D, Rerimassie VG, Srinivas KR, Zhang W (eds) GEST deliverable 5.2. Ethics debates on synthetic biology in the three regions

SSBRN Task Force (2012) Report of the task force on synthetic biology and systems biology resource network. Planning Commission, New Delhi

Stemerding D, Rerimassie VG (2013) Discourses on synthetic biology in Europe. Rathenau Instituut, The Hague

Stilgoe J (2014) Don't shut the door on the synthetic biology debate. The Guardian, 8 May. http://www.theguardian.com/science/political-science/2014/may/08/dont-shut-the-door-onthe-synthetic-biology-debate Accessed 2 June 2014

Synenergene (2014) What is Synenergene? http://synenergene.eu/information/what-synenergene Accessed 2 June 2014

Tait J (2009) Governing synthetic biology: processes and outcomes. In: Schmidt M, Kelle A, Ganguli-Mitra A, De Vriend H (eds) Synthetic biology: the technoscience and its societal consequences. Springer, New York, Heidelberg, pp 144-154

ter Meulen V (2014) Time to settle the synthetic controversy. Nature: 509(135). doi:10.1038/509135a

TESSY (2008) Documentation of TESSY stakeholder workshop Brussels, 10 June 2008. Opportunities for stakeholders. TESSY deliverable D3.2

Torgersen H, Schmidt M (2013) Frames and comparators: how might a debate on synthetic biology evolve? Futures 48(100):44-54. doi:10.1016/j.futures.2013.02.002

UNESCO (2006) Universal declaration on bioethics and human rights. UNESCO, Paris

Van Est R, Stemerding D (2012) Making perfect life: European governance challenges in 21 st century bio-engineering. Science and Technology Options Assessment, Brussels

Yang H (2011) Actively promote the development of synthetic biology. Academic division of China association of science and technology, Ethical issues of synthetic biology and biosafety. China Science and Technology Press, Beijing, pp 18-21 
Zhai X, Renzong Q (2010) Ethics and regulation of synthetic biology. Science Times 2010-7-16 A3. http://news.sciencenet.cn/htmlnews/2010/7/234644.shtm. Accessed 12 June 2014

Zhang J, Marris C, Rose N (2011) The transnational governance of synthetic biology: scientific uncertainty, cross-borderness and the 'art' of governance. BIOS, London School of Economics and Political Science, London

Zhang W (2014) Discourses on synthetic biology in China. In: Stemerding D, Rerimassie VG, Srinivas KR, Zhang W (eds) GEST deliverable 5.2. Ethics debates on synthetic biology in the three regions 